\title{
The role of involuntary aware memory in the implicit stem and fragment completion tasks: A selective review
}

\author{
SACHIKO KINOSHITA \\ Macquarie University, Sydney, New South Wales, Australia
}

\begin{abstract}
In this article I argue that an awareness of the study episode that arises involuntarily during an implicit stem/fragment completion test can under some conditions lead to enhanced repetition priming effects, even though subjects are not engaged in intentional retrieval. I review findings that are consistent with this possibility, which include the effects of depth of processing, and of typography match and new association priming following deep encoding. A theoretical account of involuntary aware memory couched within Moscovitch's (1995b) memory systems framework which suggests that the medial-temporal lobe/hippocampal (MTL/H) complex functions as a memory module is outlined. A putative mechanism is proposed in which involuntary aware memory of a studied item enhances the size of repetition priming effects by guiding its selection in preference to the competitors.
\end{abstract}

The last decade has seen an explosion of research on implicit memory. The main reason for this sustained interest in implicit memory must be attributed to the fact that it is dissociated from explicit memory. This is most strikingly demonstrated by amnesic subjects who show intact performance on tests of implicit memory yet show no awareness of having studied the test items. However, the level of aware memory is not always this low. Most researchers working with university students as subjects would be familiar with the experience of testing these subjects in an ostensibly implicit memory test, and being told during a postexperimental debriefing that they were aware that the test items were presented in a recent study episode. Does this awareness matter? Does it in fact inflate the amount of repetition priming effects?

In a review of implicit memory in normal human subjects, Roediger and McDermott (1993) addressed this question regarding the role of phenomenological awareness of memory in implicit memory tasks. They argued that awareness would not be troubling and would not compromise the implicit test, unless this awareness caused the subject to change his or her strategy and adopt intentional retrieval processes on upcoming items. This sentiment is echoed by a number of memory researchers, including Tulving, who is cited by Roediger and McDermott as saying that "your hand-wringing over how to tell a

The research reported in this article was supported by a Macquarie University Research Grant to the author. I am grateful to the editor John Wixted, and to Roddy Roediger, Barbara Knowlton, Alan RichardsonKlavehn and two anonymous reviewers for helpful suggestions and comments on earlier versions of the article. Correspondence concerning this article should be addressed to S. Kinoshita, Department of Psychology, Macquarie University, Sydney, NSW, Australia, 2109 (e-mail: sachiko.kinoshita@mq.edu.au). 'pure' implicit task, or implicit memory performance is a bit overdone. . . The issue is not that important at this early stage of the game." I think that we have now reached a stage where it is appropriate to reconsider these claims. Although the possibility that there is a role for the brain mechanism damaged in amnesia to modulate the size of repetition priming effects has been raised in previous studies (Curran \& Schacter, 1997; Curran, Schacter, \& Bessenhoff, 1996; Kinoshita \& Wayland, 1993), these were concerned with just one phenomenon (the perceptual specificity effect). These studies also did not describe a mechanism by which awareness of the study episode could affect the size of repetition priming effect without involving intentional retrieval. The aim of the present article is therefore to review a body of data suggesting a role for involuntary aware memory in modulating the amount of repetition priming effect across different manipulations, and to consider a common mechanism for its role.

Before I turn to the review, it is important to define its scope. As my title indicates, this review is selective, in two respects. First, I will discuss only studies in which the stem and fragment completion tasks have been used, because the clearest evidence of involvement of involuntary aware memory is found in these tasks; this review will not cover other data-driven implicit memory tasks such as the lexical decision task or perceptual identification task. Second, I shall be selective in the pattern of data that I review. The predominant finding in the implicit memory literature is that of a dissociation between performance on an implicit memory test and the level of awareness of the studied event, and I do not wish to give the impression that the contrary pattern reviewed here overrides the importance of the predominant pattern. Neither do I intend to challenge the widely accepted theoretical frameworks such as the transfer-appropriate processing view (Roedi- 
ger, 1990; Roediger \& Blaxton, 1987b) or the multiple memory systems view (e.g., Schacter, 1992), which do a good job of explaining the dissociative pattern. Rather, the aim of this review is to complement these accounts by drawing researchers' attention to the studies suggesting a positive relationship between involuntary aware memory and the size of repetition priming effects, and to consider a mechanism that can explain this relationship.

This review is organized as follows. First, I will summarize how involuntary aware memory is viewed within the currently predominant frameworks. I will then argue that contrary to what most frameworks suggest, involuntary aware memory should be viewed separately from what is typically interpreted as implicit or explicit memory, and defend this position within a framework of memory proposed by Moscovitch $(1992,1995 b)$. The next section is a review of empirical findings from stem/ fragment completion tasks which have suggested a role for involuntary aware memory in modulating the size of repetition priming effects. I will then describe a mechanism based on Moscovitch's (1995b) framework that explains this modulation. In the final section, I will discuss the implication of the proposed mechanism within a broader context.

\section{Involuntary Aware Memory Within Current Frameworks}

Definition of implicit memory. Definitions of implicit and explicit memory have troubled many researchers. Earlier debate has concerned the issue of separating tasks and processes-that is, whether the performance on an ostensibly "implicit" memory task reflects only implicit memory (the process-purity assumption; see Jacoby, 1991). It is now recognized that such an assumption is not necessarily warranted, and that researchers wishing to claim that their task of choice is not simply tapping explicit memory need to provide empirical checks for their claim. For a comprehensive review and specific methodologicalrecommendations, readers should refer to Roediger and McDermott (1993; see also RichardsonKlavehn \& Gardiner, 1996; Richardson-Klavehn, Lee, Joubran, \& Bjork, 1994).

Notwithstanding this agreement that an "implicit memory test" does not necessarily measure implicit memory, how implicit memory should be defined is still being debated. Researchers agree that implicit memory is memory that does not involve conscious recollection: The main issue of contention is whether conscious recollection should refer to intentional retrieval or phenomenological awareness of the study episode.

Schacter, Bowers, and Booker (1989) presented the first thorough analysis of this distinction. Intentional retrieval, in their view, refers to the act of deliberately searching for an item from the study episode. Phenomenological awareness, on the other hand, refers to the recollective experience that entails a reexperiencing of a recent episode. They considered five different scenarios in which these two meanings of conscious recollection could co- occur. The most troublesome scenario described a situation in which, in the course of completing word stem solutions, subjects' become aware that some, but not all, of the solutions that they have produced represent study list targets, but nevertheless they do not attempt to retrieve the solutions from the study list deliberately. Schacter et al. argued that it is difficult to classify this case unambiguously as an instance of either implicit or explicit memory on the basis of an awareness of the study episode, because the awareness is not all or none.

Instead, Schacter et al. (1989) argued that the implicit/ explicit distinction is better defined in terms of intentionality of retrieval, because an empirical criterion can be proposed in this case. This retrieval intentionality criterion is said to be satisfied when an experimental manipulation is found to produce a dissociation in the performance on the same task under intentional and nonintentional mode of retrieval. Within this definition, then, the scenario above, described as a case of involuntary aware memory, would be classified as an instance of implicit memory to the extent that it reflects nonintentional retrieval.

A different approach to the implicit/explicit distinction has been proposed by Jacoby and his colleagues (Jacoby, 1991, 1996; Jacoby, Toth, \& Yonelinas, 1993). According to this approach, performance on a memory test reflects the contribution of two types of influence of memory: automatic or controlled. Following the popularly held assumptions about the characteristics that distinguish between these two processes (e.g., Posner \& Snyder, 1975), automatic influence of memory is defined as unaware and unintentional, and controlled influence of memory as aware and intentional.Jacoby (1991) proposed a method referred to as the process dissociation procedure to estimate the contribution of these two types of memory influences. In this procedure, performance on a memory test such as a fragment completion task is contrasted under the inclusion and exclusion conditions. The inclusion condition instructs subjects to use the fragment as a cue to recall a studied word; in the exclusion condition, subjects are asked to use the fragment to recall a studied word but not to use studied words to complete the fragment. The assumption is that in the inclusion condition, the automatic and controlled influences of memory act in concert. In the exclusion condition, in contrast, the two types of influences of memory are placed in opposition, and hence a studied word would be produced only if it came to mind automatically (unintentionally) and subjects failed to recognize it as having been presented in the study episode. The contribution of automatic and controlled influences can then be estimated by solving simultaneous equations relating the fragment completion rates observed in the inclusion and exclusion tests with these influences of memory.

Richardson-Klavehn, Gardiner, and Java (1994), following Schacter et al. (1989), argued that intentionality of retrieval and phenomenological awareness of memory are theoretically distinguishableconcepts, and they pointed 
out that Jacoby's definition conflates the two. In their study, instead of the exclusion instruction they used the "opposition" instruction in which subjects are asked to report the first word that comes to mind but to omit the word if the word was studied. In this study, the amount of repetition priming observed under the direct, indirect, and opposition conditions was used to estimate the contribution of voluntary aware memory, involuntary unaware memory, and involuntary aware memory.

Jacoby (1998) has since responded to RichardsonKlavehn, Gardiner, and Java's (1994) criticism by emphasizing the assumptions that need to be met in applying the process dissociation procedure in order to obtain valid estimates of the contributions of automatic and controlled influences of memory. The assumptions involve those of (1) equivalence, in which the contribution of controlled influence of memory should be equivalent under the inclusion and exclusion conditions; and (2) independence, in which the automatic and controlled influences should be independent of each other. According to this approach, the opposition instruction used by Richardson-Klavehn, Gardiner, and Java would have encouraged subjects to use the generate-recognize strategy to complete the stems, which would have violated both assumptions. To put it another way, within Jacoby's framework there is no scope for the concept of involuntary aware memory, because for a word to be generated involuntarily and then subsequently to be recognized would mean that the automatic and controlled influences of memory were not independent (for an alternative analysis, see Bodner, Masson, \& Caldwell, 2000).

To summarize, implicit memory has been defined variously in terms of absence of intentional retrieval (involuntary retrieval), absence of phenomenologicalawareness (unaware memory), or absence of both intentional retrieval and phenomenological awareness (involuntary unaware memory). From the first perspective (e.g., Schacter et al., 1989), involuntary aware memory would be regarded as an instance of implicit memory insofar as there was no intention to deliberately retrieve events from the study episode; for Jacoby and his colleagues, who equate phenomenologicalawareness with intentionality, involuntary aware memory is defined out of existence as a theoretical impossibility.

In this article, I take the position put forward by Richardson-Klavehn, Gardiner, and Java (1994) that there is a distinction between phenomenological awareness and intention. This distinction makes intuitive sense. Take perception of objects, for example: No one takes exception to the claim that we perceive things in the environment without intending to do so. Literature on motor actions (e.g., Norman \& Shallis, 1986) also indicates how some actions are performed without intentional control. It should not be surprising, then, that we become aware of the fact that something is familiar without intending to do so, as in a chance encounter with an old acquaintance. Richardson-Klavehn and colleagues (e.g., Richardson-Klavehn \& Gardiner, 1996; Richardson-
Klavehn, Lee, et al., 1994) have also provided empirical support for the distinction by showing that the retrieval intentionality criterion is satisfied even when subjects in the implicit retrieval condition are unanimously aware of the study episode.

If the possibility of involuntary aware memory is to be accepted, the challenge, then, is to provide a theoretical framework for explaining how the phenomenological awareness of a study episode can be triggered without the intention to retrieve the episode. Most current frameworks of memory have not specifically addressed the case of involuntary aware memory, but there is one exception. In the next section, I will describe the framework proposed by Moscovitch (1992, 1995a, 1995b).

Moscovitch's framework of memory. Moscovitch (1992, 1995a, 1995b) emphasized the phenomenological awareness of study episode as a defining characteristic of explicit memory. According to Moscovitch, phenomenologically aware forms of memory are supported by two separate systems: an associative memory module, which is identified neuroanatomically with the medial temporal lobes/hippocampus (MTL/H) and related limbic structures, and a nonmodular, central system, which is identified with the prefrontal cortex. At retrieval, an externally or internally generated cue interacts automatically with the memory trace and reactivates it, a process described by Tulving $(1983,1985)$, as ecphory. The encoding specificity principle (Tulving \& Thomson, 1973) states that such a process is particularly likely to occur when the study and test conditions are similar. In this ecphoric process, the frontal system is responsible for intentional, strategic retrieval such as that demanded in a recall task when minimal cues are provided at retrieval (e.g., "What words did you see in this experiment?") and subjects need to generate their own retrieval cues. The MTL/H system, on the other hand, Moscovitch argued, satisfies the criteria of a module as suggested by Fodor (1983); that is, it is an information processing device that is domain specific, mandatory, and informationally encapsulated, and that has shallow output. Of particular relevance to the notion of involuntary aware memory is the feature of mandatoriness. Within the MTL/H module, intentionality is not necessary for retrieval: "Given the appropriate cue, the memory pops into mind whether we intend or not, even when we may be distracted by other activities" (Moscovitch, 1995b, p. 282).

Within this framework, the role of attention at encoding is important. There is a general consensus that, at encoding, various elements are bound together to form a coherent episodic trace, a process that is ascribed to the MTL/H system (e.g., Cohen \& Eichenbaum, 1993; Kroll, Knight, Metcalfe, Wolf, \& Tulving, 1996; McClelland, McNaughton, \& O'Reilly, 1995). There is much evidence that focused attention is necessary for this binding to take place: Studies abound in which it has been demonstrated that unattended information or information encoded under divided attention is poorly recollected in explicit memory tests (Baddeley, Lewis, Eldridge, \& Thomson, 
1984; Craik, Govoni, Naveh-Benjamin, \& Anderson, 1996; Eich, 1984; Merikle \& Reingold, 1991; Parkin, Reid, \& Russo, 1990). Moscovitch(1995a, 1995b) has argued that this consciously apprehended information is the domain of the MTL/H module: The MTL/H module binds into a memory trace the neural elements that mediate the conscious experience of the encoded event. He further suggests that when the ecphoric process retrieves the contents of the memory trace, the conscious awareness experienced at encoding is retrieved along with the other elements bound into the trace; this retrieval of a trace imbued with consciousness accounts for the felt experience of remembering, the feeling of reexperiencing the event. In this sense, the conscious awareness present at encoding is said to be "recovered."

In summary, involuntary aware memory is characterized by the two features that its name suggests: involuntariness and awareness. Recognition occurs spontaneously even though it is not specifically required by the task. It comes about under conditions conducive for observing the encoding specificity principle. I now turn to the studies of implicit memory which suggest a role for involuntary aware memory, and describe how Moscovitch's framework may be used to explain these phenomena.

\section{Empirical phenomena}

In this section, I will first describe the nature of the task used in the studies, then describe the findings that implicate the involvement of involuntary aware memory. A mechanism in which involuntary aware memory modulates the size of repetition priming effect will then be described in the following section.

Data-driven implicit memory test. Word stem and word fragment completion tasks are probably the most common tasks used to tap implicit memory, and either of these tasks was used in all the studies described below. In word stem completion, subjects are asked to report the first word that comes to mind that starts with a given three-letter stem (e.g., win-). Typically, the stems are chosen so that there are multiple solutions (e.g., window, wind, winter, winner). In word fragment completion, the fragment consists of a word with letters deleted and there is one correct solution (e.g., $-y s-e-y$ for mystery).

Roediger and Blaxton (1987b) were the first to point out that these tasks differ from tasks used to tap explicit memory such as recall and recognition not only in terms of retrieval orientation but also in terms of the type of processing required along the data-driven/conceptually driven dimension. Data-driven processes are guided by the physical information present, whereas conceptually driven processes are top-down processes guided by subjects' semantic knowledge and expectations. Examples of this distinction applied to encoding activities would be judgments of appearance (e.g., counting the T-junctions in words) versus judgments of meaning (pleasantness rating of words), elaboration (judging whether a word fits meaningfully into a sentence), or organization (grouping words according to semantic categories). More impor- tantly, Roediger and Blaxton (1987b) pointed out that tasks typically used to tap explicit memory such as recall and recognition are primarily conceptually driven, whereas tasks used to date to tap implicit memory such as word fragment completion and word stem completion are primarily data driven. From the transfer-appropriate processing principle (Kolers \& Roediger, 1984; Morris, Bransford, \& Franks, 1977), then, it would be expected that performance on free recall and recognition tasks would be enhanced by encoding activities that engage conceptually driven processes, whereas performance on word stem and word fragment completion tasks would be enhanced by encoding activities that engage data-driven processes, but not by conceptually driven encoding.

The empirical phenomena to be described, however, violate the patterns expected from the transfer-appropriate processing framework and have shown that performance on data-driven implicit memory tasks had been facilitated by manipulations known to enhance performance on recall and recognition. The three manipulations to be described are (1) depth of processing, (2) typography match, and (3) reinstatement of episodic context; further, the latter two effects are found specifically under elaborative encoding conditions. I will now turn to the studies in which these manipulations have been used, and argue that involuntary aware memory is indicated in all of these studies.

Depth-of-processing effect. From the transfer-appropriate processing perspective, because the word stem and word fragment completion tasks are considered to be primarily data-driven tasks, there is little basis to expect a depth-of-processing manipulation to enhance priming in these tasks. In the memory-intact subject population, however, over a number of studies, a small but consistent priming advantage for deeply encoded words has been reported (for reviews, see Brown \& Mitchell, 1994; Challis \& Brodbeck, 1992). Hamann and Squire (1996) reported that this depth-of-processing effect on word stem and word fragment completion tasks was reduced in amnesic subjects, and argued that conscious recollection was contributing to the levels-of-processing effect. It is not clear, however, what form this "conscious recollection" takes. As mentioned earlier, researchers have used it to refer to either intentional retrieval of studied items from memory or phenomenological awareness that the test items were encountered earlier, and it is not clear to which sense Hamann and Squire were referring.

Bowers and Schacter (1990; see also Schacter et al., 1989) reported data directly relating the depth-ofprocessing effect with awareness of the study episode. In their study, subjects studied words either under a deep encoding instruction (pleasantness rating task) or under a shallow encoding instruction (counting T-junctions in a word) and then performed a stem completion task. The subjects also completed a postexperimental questionnaire assessing the extent of their awareness of the relationship between study and test, with question items such as "What did you think was the purpose of the stem completion task that you just finished? While doing the 
stem completion test, did you notice whether you completed some of the stems with the words studied in the earlier list?" Subjects were classified as test unaware only if they responded negatively to all questions. Test-aware subjects were found to complete more stems with the studied words following the deep as opposed to the shallow encoding condition, but the test-unaware subjects did not show this depth-of-processing effect. Bowers and Schacter argued that although there was a possibility that the test-aware subjects were engaged in intentional retrieval, given the experimental dissociations observed in previous studies between direct and indirect modes of retrieval in this task, this was unlikely.

That this depth-of-processing effect in stem completion reflects involuntary aware memory receives further support from Richardson-Klavehn, Gardiner, and Java (1994). Subjects in that study were given the word stem completion task, under three retrieval instructions: direct, indirect, and opposition. In the opposition condition, subjects were instructed to write down the first word that came to mind, but to write down a different word if the word that came to mind first had been studied. The researchers reasoned that facilitatory priming (i.e., a higher stem completion rate for studied words relative to the baseline) would be observed under this condition only if involuntary unaware influences of memory outweighed the involuntary aware influences of memory and of intentional retrieval. Facilitatory priming was observed following a shallow encoding condition, whereas inhibitory priming (lower stem completion rate for studied words relative to baseline) was observed following a deep encoding condition. In contrast, in the indirect condition, the amount of priming did not differ significantly following the deep and shallow encoding tasks. Richardson-Klavehn, Gardiner, and Java concluded that the two encoding activities produced equivalent involuntary influences of memory, but the deep encoding task produced a greater amount of involuntary aware memory.

Like Bowers and Schacter (1990), Richardson-Klavehn, Gardiner, and Java (1994) argued that subjects were not deliberately searching for the studied words that started with the given stems. For one thing, the depth-ofprocessing effect was greater in the direct retrieval condition than in the indirect retrieval condition, satisfying the retrieval intentionality criterion. Second, subjects in the indirect and opposition conditions were both instructed to give the first item that came to mind in response to each stem. Given that the stems were chosen to have multiple completions and therefore to be easy to produce a solution, Richardson-Klavehn, Gardiner, and Java argued that an intentional search for the studied items would have been strategically inefficient. Furthermore, in a postexperiment interview, although all subjects in the indirect retrieval condition stated that they had realized that some of the stems in the completion task could have been completed with the studied items, none claimed to have engaged in intentional search for the studied items. Richardson-Klavehn and Gardiner (1998) also reported that stem completion latencies were faster under the implicit retrieval instruction than under the control condition in which there was no study phase (and in which latencies were in turn faster than under the intentional retrieval instruction). These observations therefore strongly suggest that the depth-of-processing effect on word stem completion under the indirect retrieval instruction does not reflect intentional retrieval. ${ }^{1}$

Typography-match effect following deep encoding. The typography-match effect refers to a greater priming effect observed when the study and test typographies match. Roediger and Blaxton (1987a) were the first to report the finding by using word fragment completion and interpreted the effect in terms of enhanced overlap in data-driven processing. Kinoshita and Wayland (1993) investigated the effect with amnesic subjects. Moderately amnesic Korsakoff patients and age-matched controls studied typed and handwritten words in a deep encoding (liking rating) task and later completed word fragments in a typography that either matched or did not match the study typography. A statistically significant typography-match effect was observed only with handwritten words and was limited to the control subjects. Furthermore, the amount of typography-specific priming effect was correlated with the subject's score on the Wechsler Memory Scale. These results are consistent with the possibility that the typography-specific priming is associated with some aspects of explicit memory. The interpretation offered by Kinoshita and Wayland at the time was that the original study episode is "more easily recaptured" when the study and test typographies match, particularly when handwritten words are used that are not like preexperimental encounters with the words. If this recollection of the original study episode does occur involuntarily (although there is nothing in the study that argues either for or against this), the typography-match effect found with controls but not with amnesics may be considered an instance of involuntary aware memory.

This involuntary-aware-memory interpretation of the typography-match effect was specifically investigated in a study reported by Curran et al. (1996), who used uppercase and lowercase words in stem completion. In a review of previous findings, they noted that the typographymatch effects were sometimes enhanced by conditions yielding high levels of explicit memory. In line with this observation, they found typography-match effects following a deep encoding task (liking rating task) when the study-test retention interval was short and when the study presentation was repeated. Interestingly, the typography match effect was not found under the same task parameters when subjects were instructed to search for the studied words from memory that fit the stem. Given these results, Curran et al. argued that the observed typographymatch effect reflected involuntary aware memory in which unique study episodes are brought to awareness even though the subject is not trying to retrieve these episodes. ${ }^{2}$

Though not interpreted by the authors as such, a finding by Jacoby, Levy, and Steinbach (1992) may also be 
considered as reflecting involuntary aware memory. These researchers measured the extra facilitation in reading time observed as a result of prior exposure to a short passage when the typography was kept constant versus when it was changed. They reported a curious finding in which a typography-match effect was not obtained when subjects read the passage aloud (which was assumed to force processing to occur in a word-by-word fashion and hence emphasize data-driven processing) but when subjects read the passage silently to answer comprehension questions (which was expected to involve more conceptually driven processing). Jacoby et al. (1992) suggested that typography-match effects are found in reading connected passages when "reading is backgrounded in the service of a semantic task" (p. 15), but they did not provide a theoretical basis for this claim. One possibility that can be suggested is that by emphasizing comprehension, the first reading episode was encoded more deeply and hence this episode was likely to come to mind involuntarily upon rereading. This recapturing of the study episode may have been further enhanced by a match in study and test typography.

New association priming effect following deep encoding. Graf and Schacter $(1985,1987)$ pioneered the procedure for investigating priming for novel associations in word stem completion. In their paradigm, subjects study pairs of words (e.g., officer-window) and are later asked to complete word stems, each accompanied with a context word. Subjects are more likely to complete the stem with a studied word when the stem is accompanied by the same word as that with which it was paired (e.g., officer-win-) than when the stem is paired with a different word (e.g., castle-win-). The facilitative effect of reinstating context is referred to as the new association priming effect (or "context-dependent priming for new associations"; Graf $\&$ Schacter, 1985).

In the stem completion task, new association priming is found reliably only following a semantic encoding task. Graf and Schacter (e.g., 1987), however, argued that the new association priming effect does not reflect a deliberate search for the studied word. This claim is based on a number of functional dissociations observed under direct and indirect retrieval modes; that is, it satisfies the retrieval intentionality criterion. Factors that affect performance on the direct, but not the indirect, test include the type of elaborative encoding (e.g., generating a sentence that links word pairs vs. rating a sentence containing word pairs for meaningfulness; Schacter \& Graf, 1986) and retroactive and proactive interference manipulations (Graf \& Schacter, 1987). On the other hand, a match of modality (auditory vs. visual) between study and test has been found to have an effect under indirect, but not direct, retrieval instructions.

Although these studies established that the new association priming effect does not reflect intentional retrieval, there is evidence that it is modulated by the level of awareness of the study episode. Using a postexperimental questionnaire, Bowers and Schacter (1990; see also Schacter et al., 1989) reported that the new association priming effect was greater in test-aware subjects than in test-unaware subjects. Reingold and GoshenGottstein (1996) also showed that the facilitative effect of reinstating the context in stem completion is eliminated under the exclusion instruction, arguing that the new association priming effect reflects controlled (aware) influences of memory. In addition, amnesic patients do not show new association priming effects reliably (Mayes \& Gooding, 1989; Shimamura \& Squire, 1989). ${ }^{3}$ Taken together, the new association priming effect in stem completion appears to reflect involuntary aware memory.

Summary. Common among these studies involving the effects of depth of processing, typography match, and new association priming is that these phenomena are associated with enhanced repetition priming effects. These manipulations are also known to enhance performance on explicit tests of memory. At the same time, however, these studies also provide evidence that subjects were not engaged in intentional retrieval. As such, the enhanced repetition priming effects observed in these studies may be best described as reflecting involuntary aware memory. I will now propose a mechanism that explains how these manipulations modulate the size of the repetition priming effects.

\section{Mechanism for Enhancing Repetition Priming Effects}

In brief, the mechanism that I propose here suggests that when there are competing lexical representations, spontaneous recognition of the studied item that occurs in response to a test cue focuses attention on it and consequently guides the lexical search process so that the studied item is processed preferentially. In detail, the mechanism is as follows.

Within the transfer-appropriate processing framework that classifies tasks along the conceptual-perceptual continuum, the word stem/fragment completion task is regarded as primarily a perceptual (data-driven) task. The work of Nelson and colleagues (e.g., Nelson, Canas, Bajo, \& Keelan, 1987) suggests further that these tasks involve a data-driven lexical search process, as attested by the effects of lexical factors such as fragment cue-target strength (i.e., baseline completion rate) and lexical set size (i.e., the number of words that share the fragment). Their PIER (processing of implicit and explicit representations) model (Nelson, Schreiber, \& McEvoy, 1992) suggests that in an implicit stem/fragment completion task, the test cue (stem or fragment) initially activates multiple candidates on the basis of orthographic information. These candidates are then sampled for verification, one at a time. I suggest that that awareness of the study episode plays a role at this stage of sampling.

On the basis of Moscovitch's (1995a, 1995b) framework, I suggest that when a word stem cue interacts with the stored memory trace (i.e., ecphory occurs) and a studied word is recognized as having been encountered recently, the perceptual (and conceptual) records of the 
original conscious experience are retrieved. Moscovitch (1995a, 1995b) emphasized the idea that awareness always has an object: To be aware necessarily implies that one is aware of something. Furthermore, this phenomenological awareness is selective. To be consciously aware of something means that it takes priority over similar, competing representations: To take an oft cited example, one can be consciously aware of only one interpretation of the Necker cube; one cannot simultaneously entertain two competing interpretations. In a stem/fragment completion task, then, the recovered neural elements that underlie the initial conscious apprehension of the event function as an attractor to facilitate the sampling of the studied word over other activated lexical representations, enhancing its production as a response.

To reiterate, in an implicit stem/fragment completion task, the test cue (the stem) initially activates multiple candidates. When the conditions are conducive (as guided by the encoding specificity principle) and the test cue triggers involuntary recognition of the studied word, the studied word is brought into the focus of attention. This means that a recognized studied word will be sampled before the competitors (other activated lexical candidates), resulting in enhanced repetition priming effects relative to when no spontaneous recognition has occurred.

Evidence of preferential processing. The way in which a spontaneously recognized word attracts attention and blocks processing of competitors may be likened to the way in which "blockers" hinder access to the desired word in word retrieval paradigms, notably in tip-of-thetongue states (see Brown, 1991, for a review of the tipof-the-tongue experience). These blockers (also called "persistent alternates" by Burke, MacKay, Worthley, \& Wade, 1991; or "interlopers" by Jones, 1989) are words related (either semantically or phonologically or both) to the target word that come repeatedly and involuntarily to mind, such as the word charity given the definition for chastity. In line with this, in an implicit fragment completion task, Smith and Tindell (1997) reported that when a word (e.g., ANALOGY) that is orthographically similar to the solution of the test fragment (e.g., ALLERGY, which is the solution to the fragment A-L-GY) had been studied, this reduced the fragment completion rate relative to the control condition in which a word unrelated to the test fragment (e.g., UNICORN) was studied. They showed that this effect (which they called the memory block ef$f e c t$ ) was not eliminated by a number of manipulations designed to discourage subjects from deliberately retrieving the study episode, such as informing subjects of the negative consequence of retrieving the studied words from memory, or using only the test fragments that could not be completed by the studied words. They took this as evidence that the memory block effect occurred as a result of involuntary retrieval of the study episode. We (Kinoshita $\&$ Towgood, in press) found that the memory block effect was reduced by manipulations that reduced the level of recognition of the studied words, such as dividing attention at study and interpolating a retention interval filled with verbal activity, indicating that the memory block effect depended on the awareness of the study episode. These findings show that involuntary aware memory is involved in countering interference from competitors that are activated by the test cues.

More direct evidence that spontaneous recognition of a studied item results in preferential sampling can be found in Kinoshita's (1999) investigation of the new association priming effect. Subjects studied pairs of words under an elaborative encoding condition (generating sentences that linked the word pairs) and then completed word stems that were paired with the original context words or recombined with different context words. In an experiment in which subjects were instructed to report all words that came to mind in response to the stem cue, they were more likely to output the studied word earlier when the context was reinstated than when the context was different from study. This result therefore demonstrates that preferential processing of studied items accompanied by the same context was responsible for the new association priming effect.

Effects of dividing attention at test. One manipulation commonly used to test whether a task is performed automatically is the use of a concurrent task to divide attention at test. Dividing attention at test has been reported to have little effect on recognition memory tests (Baddeley et al., 1984; Craik et al., 1996; Moscovitch, 1994). This finding has been taken as suggesting that the triggering of aware memory does not demand attentional resources. This in turn suggests that the modulation of repetition priming effects by involuntary aware memory should also be unaffected by dividing attention at test. This prediction was supported by the observation reported by Kinoshita and Towgood (in press) that the memory block effect in a fragment completion task (caused by prior exposure to orthographically similar words) was not affected by dividing attention at test. In contrast, however, Kinoshita (1999) found that dividing attention at test abolished the new association priming effect. How could this discrepancy be reconciled?

There are at least two reasons why dividing attention at test affected the new association priming effect in the stem completion task, contrary to what is expected from the view that dividing attention at test should not affect involuntary retrieval. One is related to the attentional requirement of the task itself. Winocur, Moscovitch, and Stuss (1996) reported that the size of the repetition priming effect in a stem completion task was reduced in the elderly, relative to young subjects, and that it was correlated with tests of frontal lobe function such as the word fluency test (FAS) and the Wisconsin Card Sorting Test (WCST). They took this as evidence that in the stem completion task, despite being implicit, controlled processes are involved in searching the lexicon for a word beginning with the stem. Dividing attention at test may have interfered with this controlled aspect of the task, rather than the involuntary retrieval of the studied item. The second reason is related to the nature of the cue that 
triggers the memory of the study episode. The new association priming effect in stem completion is conceptually mediated. That is, when the context is reinstated at test (e.g., officer-win- is presented at test when officerwindow has been studied), it facilitates not the retrieval of the memory of the studied target (window), but the memory of the context-target ensemble (officer-window) that has been unitized as a result of elaborative (conceptual) processing. There is evidence that conceptual priming effects, relative to perceptual priming effects, are more prone to effects of divided attention (e.g., Mulligan \& Hartman, 1996). In contrast, in recognition tests, the retrieval cue directly reinstates the original perceptual experience. These two factors may explain why dividing attention at test did not reduce the level of recognition memory performance, but did reduce the size of the new association priming effect.

\section{Relationship to Existing Conceptual Frameworks}

In this section, I will discuss the implications of the proposed mechanism of modulation of repetition priming effects by involuntary aware memory within a broader context. The issues to be discussed concern the roles of (1) interference and constraint, and (2) deep encoding.

Interference and constraint. The idea that interference from competitors affects the size of repetition priming effects as suggested in the proposed mechanism has its origins in a chapter by Moscovitch (1984), a precursor to his memory systems framework (Moscovitch, 1995a, 1995b). In a review of preserved learning capacity in amnesia, Moscovitch (1984) described situations likely to lead to demonstrations of preserved learning. He suggested that amnesics would show savings on tasks that satisfied the following three conditions: (1) The tasks must be so highly structured that the goal of the task and the means to achieve it were apparent; (2) the means to achieve the goal must be available to the subject (i.e., the response and strategies used to arrive at the goal would already be in the subject's repertoire); and (3) success in achieving the goal could be had without reference to a particular postmorbid event or episode.

It can be seen that the third of these conditions describes what is now called implicit (indirect) memory tasks. The second condition essentially requires that the perceptual modules that are assumed to support repetition priming are intact. The condition most relevant to the discussion at hand is the first. Data-driven implicit memory tasks generally satisfy this condition: The test cues and instructions provide the constraint necessary for one to achieve the goal (e.g., "Write down the first word that comes to mind that starts with these three letters"). However, these factors may not completely constrain performance on the studied item in a stem/fragment completion task. For example, test stems typically have multiple solutions: Although a studied word is a solution, it is not the only solution possible. Furthermore, target words are usually chosen so that of the possible comple- tions for a given stem the target is not the most likely completion (i.e., the word with the highest baseline completion rate), in order to avoid ceiling effects in observing a priming effect. Even in word fragment completion where the fragments have unique solutions, during the search of the orthographic lexicon to find a matching word, other orthographically similar candidates may be encountered, as is evidenced by the memory block effect (Smith \& Tindell, 1997) described above. This means that the size of the repetition priming effect is dependent on the interference that it receives from other potential completions during the search for a solution: The more the studied item can overcome the interference that it faces from competitors, the greater the amount of priming that will be observed.

Deep encoding. One final issue that needs to be addressed here concerns the role of depth-of-processing. In all the instances of involuntary aware memory described above, deep encoding is involved. The effects of depth of processing on standard tests of explicit memory such as recall and recognition are well known (cf. Craik \& Lockhart, 1972; Craik \& Tulving, 1975). The findings described in the present article indicate that semantic encoding also enhances the level of aware memory even when retrieval is involuntary. The question is, why? Why does deep encoding enhance the level of aware memory?

This issue is important because it is an issue that has not yet been addressed adequately by the transfer-appropriate processing framework in explaining amnesic performance on explicit memory tests (cf. Roediger, 1990). Given the assumption that recall and recognition tests tap conceptually driven processes, proponents of the transferappropriate processing approach have attempted to explain the poor amnesic performance on explicit memory tests by arguing that amnesics are impaired in conceptually driven processing (e.g., Blaxton, 1992). However, this claim runs counter to the fact that amnesics show preserved priming effects in implicit conceptual memory tests such as category generation (e.g., Gardner, Boller, Moreines, $\&$ Butters, 1973): Only when memory is tested explicitly do amnesics perform poorly in conceptually driven tests (see Cermak, Verfaellie, \& Chase, 1995). That amnesics fail to "take advantage of semantic encoding to improve their level of explicit retrieval" (Carlesimo, Marfia, Loasses, \& Caltagirone, 1996, p. 791) only describes this deficit, it does not explain it. So what is it about semantic encoding that affects normal and amnesic individuals differently in an explicit memory test?

It is worth noting that the levels-of-processing framework, as originally conceptualized, emphasized the idea that semantic encoding results in easier retrieval, irrespective of the intention to remember (Craik \& Lockhart, 1972). In Moscovitch's (1995b) words, "one cannot help but remember what one has processed deeply" (p. 282). One reason for this may be that semantic processing is inherently organizational: Because the meaning of a word belongs to the whole word, rather than to its components, semantic processing is more effective in binding the 
components together relative to other encoding tasks (e.g., Whittlesea \& Cantwell, 1987). ${ }^{4}$ A higher level of awareness of the study episode may then be viewed as a natural by-product of this integrative property of semantic encoding.

The idea that deep encoding facilitates binding explains why amnesic patients do not benefit from deeper encoding in explicit memory tests (Carlesimo et al., 1996; Cermak \& Reale, 1978), because the damage to the MTL/H system is known to result in impairment in binding. The idea also explains why amnesics perform normally on implicit conceptually driven memory tests but not on explicit tests, and why a larger difference in the level of performance between amnesics and normals is found in explicit memory tests following deep, relative to shallow, encoding.

It is important to note one caveat for the claim that semantic processing is inherently integrative. Recently, Richardson-Klavehn and Gardiner (1998) put forward the view that the depth-of-processing effects on perceptual implicit memory tests reflect lexical processing, rather than conceptual processing. They pointed out that the typical depth-of-processing manipulation involved a shallow encoding task that focused on individual letters in a word (e.g., counting T-junctions or enclosed spaces) and a deep encoding task that required the processing of a whole word (e.g., rating the pleasantness of the referent of a word). When they compared a nonsemantic encoding task that nevertheless required the processing of a word as a whole (counting syllables of a word) with a semantic encoding task (pleasantness rating), no depth-of-processing effect was found on the amount of priming. They therefore took this finding as evidence that lexical, rather than semantic, processing enhances involuntary retrieval.

The present claim is not incompatible with this view. The main tenet of the present claim is that the deep encoding tasks used in standard depth-of-processing manipulation have been more integrative than the shallow encoding tasks, and that this integrative property enhances involuntary retrieval. In this sense, the present view is entirely in agreement with the view put forward by Richardson-Klavehn and Gardiner (1998). Currently, no data suggest that semantic processing over and above lexical processing provides the "glue" that binds the components together. The present claim is therefore agnostic with regard to whether lexical or semantic processing is critical for the depth-of-processing effects on repetition priming.

\section{Conclusion and Future Directions}

Summary. In this article, I have reviewed studies which suggest that the size of the repetition priming effect is modulated by involuntary aware memory. These include manipulations of levels of processing, and of typography match and new association priming following deep encoding. The concept of involuntary aware memory does not easily fit within the predominant frameworks of memory that view implicit and explicit memories as a dichotomy, but it is accommodated by Moscovitch's (1995a, $1995 b$ ) multiple memory systems framework. This framework assumes that the MTL/H module is responsible for encoding consciously apprehended events, and it suggests that the phenomenological awareness of the study episode arises automatically as a result of an interaction between the stored memory trace and retrieval cues (ecphory). The modulation of the amount of repetition priming by involuntary aware memory may be explained within this framework as follows. When a studied episode is retrieved (involuntarily) by the MTL/H system, the subject's attention that was necessary for encoding the event is again brought into focus on the item. Under conditions in which the test task does not fully constrain the course of processing, this focused attention guides the selection of the studied item in preference to the competing representations, resulting in a greater amount of priming for the item.

In the remainder of this article, I raise some unresolved issues and future directions. These will include (1) the condition under which the awareness of the study episode is decoupled from the amount of repetition priming effects, and (2) neuropsychological implications.

When does aware memory modulate repetition priming effects? Perhaps the most pressing issue is to delineate the specific factors that produce the coupling between the level of awareness of the study episode and the size of repetition priming effects. After all, many studies on implicit memory have shown dissociation between the two: In many cases, amnesic subjects have been observed to produce just as much priming as have memoryintact subjects, indicating that the awareness of the study episode makes little contribution to the level of performance on an implicit memory test. So, under what conditions is involuntary aware memory likely to inflate the size of repetition priming effects?

As already discussed under the section on Interference and Constraint, the notion of task constraint proposed by Moscovitch (1984) can be used as a guide as we consider this issue. The modulation of the size of repetition priming effects was explained in terms of interference from competing representations present at test. It is important to note that interference here refers to functional, rather than nominal, potential for interference. For example, in stem completion, a word with a high baseline completion rate (bri-bright) is likely to have few viable competitors at test when it is the primed (studied) word, because its activation level is likely to be boosted by priming above all the competitors. In contrast, another word with the same stem but a lower baseline rate (e.g., bri-brim) is likely to experience more interference at test, because, even with priming, its activation level may not be as high as the baseline rates of other completions. This suggests that even under identical study and test conditions, the extent of coupling between awareness of the study episode and the amount of repetition priming can vary, depending 
on the baseline completion rate. Specifically, an awareness of the study episode is predicted to play a greater role in enhancing the size of the repetition priming effect when the baseline completion rate is low as opposed to when it is high. There are factors known to affect the baseline completion rates (see Graf \& Williams, 1987), such as word frequency, word length, and meanings per word. Future studies could be directed usefully toward testing the prediction above, manipulating these factors while holding all other variables constant.

It is also important to note that the mechanism proposed in this article applies to the stem and fragment completion task but that it is currently unclear how it would apply to other data-driven implicit memory tasks such as the perceptual identification task and the lexical decision task. The proposed mechanism assumes a lexical search mechanism in which a cue provided at test (e.g., word stem/ fragment) initially activates several lexical candidates, followed by a one-at-a-time sampling of the candidates. Intuitively, it seems likely that the lexical search processes involved in the perceptual identification task are more tightly constrained by the stimulus cues present, and hence there is less scope for involuntary aware memory to play a role. The finding reported by Schacter and Church (1992), indicating that voice-specific priming was observed in the auditory stem completion task, but not in the auditory perceptual identification task, is consistent with this possibility. Although the lexical decision task also appears to be constrained by the stimulus cues present, this task is considered by many visual word recognition researchers to tap the familiarity of the target (Balota \& Chumbley, 1984; Forster, 1981). Thus, like the stem and fragment completion task, this task too may be sensitive to aware forms of memory, but through a different mechanism. A more definitive conclusion regarding the role of involuntary aware memory in other data-driven implicit memory tests must therefore await future investigation.

Neuropsychological implications. In the present framework, involuntary aware memory is identified neuroanatomically with the MTL/H complex. It is well known that other structures, such as the prefrontal cortex, can cause memory deficits either in addition to or instead of the MTL/H complex. One possibility suggested by this is that the relationship described here between the level of aware memory and the amount of repetition priming effects may not be observed for patients whose damage is located outside the MTL/H complex. For example, amnesics whose damage is located solely in the frontal lobes and not in the MTL/H complex may show the typographymatch effect following semantic encoding, just like normal subjects. The fact that these patients generally show less impaired recognition relative to recall (e.g., Glisky, Polster, \& Routhieaux, 1995; Shimamura, Janowsky, \& Squire, 1990) adds strength to this prediction, since it is generally accepted that recognition may be based on a less voluntary form of retrieval than recall (e.g., Moscovitch, 1992), suggesting that involuntary retrieval may be relatively spared in the purely frontal amnesics. These and other possibilities await future investigation.

\section{REFERENCES}

Baddeley, A., Lewis, V., Eldridge, M., \& Thomson, N. (1984). Attention and retrieval from long-term memory. Journal of Experimental Psychology: General, 113, 518-540.

Balota, D. A., \& Chumbley, J. I. (1984). Are lexical decisions a good measure of lexical access? The role of word frequency in the neglected decision stage. Journal of Experimental Psychology: Learning, Memory, \& Cognition, 10, 340-357.

Blaxton, T. A. (1992). Dissociations among memory measures in memory-impaired subjects: Evidence for a processing account of memory. Memory \& Cognition, 20, 549-562.

Bodner, G. E., Masson, M. E. J., \& CAldwell, J. I. (2000). Evidence for a generate-recognize model of episodic influences on word-stem completion. Journal of Experimental Psychology: Learning, Memory, \& Cognition, 26, 267-293.

Bowers, J. S., \& Schacter, D. L. (1990). Implicit memory and test awareness. Journal of Experimental Psychology: Learning, Memory, \& Cognition, 16, 404-416.

Brown, A. S. (1991). A review of the tip-of-the-tongue experience. Psychological Bulletin, 109, 204-223.

Brown, A. S., \& Mitchell, D. B. (1994). A reevaluation of semantic versus nonsemantic processing in implicit memory. Memory \& Cognition, 22, 533-541.

Burke, D. M., MacKay, D. G., Worthley, J. S., \& Wade, E. (1991). On the tip of the tongue: What causes word finding failures in young and older adults? Journal of Memory \& Language, 30, 542-579.

Carlesimo, G. A., Marfia, G. A., Loasses, A., \& Caltagirone, C. (1996). Perceptual and conceptual components in implicit and explicit stem completion. Neuropsychologia, 34, 785-792.

Cermak, L. S., Blackford, S. P., O'Connor, M., \& Bleich, R. P. (1988). The implicit memory ability of a patient with amnesia due to encephalitis. Brain \& Cognition, 7, 145-156.

Cermak, L. S., \& Reale, L. (1978). Depth of processing and retention of words by alcoholic Korsakoff patients. Journal of Experimental Psychology: Human Learning \& Memory, 4, 165-174.

Cermak, L. S., Verfaellie, M., \& Chase, K. A. (1995). Implicit and explicit memory in amnesia: An analysis of data-driven and conceptuallydriven processes. Neuropsychology, 9, 281-290.

Challis, B. H., \& Brodbeck, D. R. (1992). Level of processing affects priming in word fragment completion. Journal of Experimental Psychology: Learning, Memory, \& Cognition, 18, 595-607.

Cohen, N. J., \& Eichenbaum, H. (1993). Memory, amnesia, and the hippocampal system. Cambridge, MA: MIT Press.

Craik, F. I. M., Govoni, R, Naveh-Benjamin, M., \& Anderson, N. D. (1996). The effects of divided attention on encoding and retrieval processes in human memory. Journal of Experimental Psychology: General, 125, 159-180.

Craik, F. I. M., \& Lockhart, R. S. (1972). Levels of processing: A framework for memory research. Journal of Verbal Learning \& Verbal Behavior, 11, 671-684.

Craik, F. I. M., \& Tulving, E. (1975). Depth of processing and the retention of words in episodic memory. Journal of Experimental Psychology: General, 104, 268-294.

Curran, T., \& Schacter, D. L. (1997). Implicit memory: What must theories of amnesia explain? Memory, 5, 37-48.

Curran, T., Schacter, D. L., \& Bessenhoff, G. (1996). Visual specificity effects on word stem completion: Beyond transfer appropriate processing? Canadian Journal of Experimental Psychology, 50, 2233.

EICH, E. (1984). Memory for unattended events: Remembering with and without awareness. Memory \& Cognition, 12, 105-111.

Fodor, J. (1983). The modularity of mind. Cambridge, MA: MIT Press, Bradford Press.

Forster, K. I. (1981). Frequency blocking and lexical access: One mental lexicon or two? Journal of Verbal Learning \& Verbal Behavior, 20, 190-203. 
GARDINER, J. M. (1988). Functional aspects of recollective experience. Memory \& Cognition, 16, 309-313.

Gardner, H., Boller, F., Moreines, J., \& Butters, N. (1973). Retrieving information from Korsakoff patients: Effects of categorical cues and reference to the task. Cortex, 9, 165-175.

Glisky, E. L., Polster, M. R., \& Routhieaux, B. C. (1995). Double dissociation between item and source memory. Neuropsychology, $\mathbf{9}$, 229-235

GraF, P., \& Schacter, D. L. (1985). Implicit and explicit memory for new associations in normal and amnesic patients. Journal of Experimental Psychology: Learning, Memory, \& Cognition, 11, 501-518.

GRAF, P., \& Schacter, D. L. (1987). Selective effects of interference on implicit and explicit memory for new associations. Journal of Experimental Psychology: Learning, Memory, \& Cognition, 13, 45-53.

Graf, P., \& Williams, D. (1987). Completion norms for 40 three-letter word stems. Behavior Research Methods, Instruments, \& Computers, 19, 422-445.

Hamann, S. B., \& Squire, L. R. (1996). Level-of-processing effects in word-completion priming: A neuropsychological study. Journal of Experimental Psychology: Learning, Memory, \& Cognition, 22, 933947

JACOBY, L. L. (1991). A process dissociation framework: Separating automatic from intentional uses of memory. Journal of Memory \& Language, 30, 513-541.

JACOBY, L. L. (1996). Dissociating automatic and consciously controlled effects of study/test compatibility. Journal of Memory \& Language, 35, 32-52.

JACOBY, L. L. (1998). Invariance in automatic influences of memory: Toward a user's guide for the process-dissociation procedure. Journal of Experimental Psychology: Learning, Memory, \& Cognition, 24, 3-26.

JAcoby, L. L., Levy, B. A., \& Steinbach, K. (1992). Episodic transfer and automaticity: Integration of data-driven and conceptually-driven processing in rereading. Journal of Experimental Psychology: Learning, Memory, \& Cognition, 18, 15-24.

JACoby, L. L., Tотн, J. P., \& Yonelinas, A. P. (1993). Separating conscious and unconscious influences of memory: Measuring recollection. Journal of Experimental Psychology: General, 122, 139-154.

JonEs, G. V. (1989). Back to Wordsworth: Role of interlopers in the tipof-the-tongue phenomenon. Memory \& Cognition, 17, 69-76.

Kinoshita, S. (1999). Priming for novel associations: Evidence for an attentional component. Memory, 7, 385-404.

Kinoshita, S., \& Towgood, K. (in press). The effects of dividing attention on the memory block effect. Journal of Experimental Psychology: Learning, Memory, \& Cognition.

Kinoshita, S., \& WAYLAND, S. V. (1993). Effects of surface features on word-fragment completion in amnesic subjects. American Journal of Psychology, 106, 67-80.

Kolers, P. A., \& Roediger, H. L., III (1984). Procedures of mind. Journal of Verbal Learning \& Verbal Behavior, 23, 425-449.

Kroll, N. E. A., Knight, R. T., Metcalfe, J., Wolf, E. S., \& TulvING, E. (1996). Cohesion failure as a source of memory illusions. Journal of Memory \& Language, 35, 176-196.

Marsolek, C. J., Kosslyn, S. M., \& Squire, L. R. (1992). Formspecific visual priming in the right cerebral hemisphere. Journal of Experimental Psychology: Learning, Memory, \& Cognition, 18, 492-508.

Mayes, A. R., \& Gooding, P. (1989). Enhancement of word completion priming in amnesics by cueing with previously novel associates. Neuropsychologia, 27, 1057-1072.

McClelland, J. L., McNaughton, B. L., \& O'Reilly, R. C. (1995). Why there are complementary learning systems in the hippocampus and neocortex: Insights from the successes and failures of connectionist models of learning and memory. Psychological Review, 102, 419-437.

Merikle, P. M., \& Reingold, E. M. (1991). Comparing direct (explicit) and indirect (implicit) measures to study unconscious memory. Journal of Experimental Psychology: Learning, Memory, \& Cognition, 17, 224-233.

Morris, C. D., Bransford, J. D., \& Franks, J. J. (1977). Levels of processing versus transfer-appropriate processing. Journal of Verbal Learning \& Verbal Behavior, 16, 519-533.
Moscovitch, M. (1984). The sufficient conditions for demonstrating preserved memory in amnesia: A task analysis. In L. R. Squire \& N. Butters (Eds.), The neuropsychology of memory (pp. 104-114). New York: Guilford Press.

Moscovitch, M. (1992). Memory and working-with-memory: A component process model based on modules and central systems. Journal of Cognitive Neuroscience, 4, 257-267.

Moscovitch, M. (1994). Cognitive resources and dual-task interference effects at retrieval in normal people: The role of the frontal lobes and medial temporal cortex. Neuropsychology, 8, 524-534.

Moscovitch, M. (1995a). Models of consciousness and memory. In M. S. Gazzaniga (Ed.), The cognitive neurosciences (pp. 1341-1356). Cambridge, MA: MIT Press, Bradford Books.

Moscovitch, M. (1995b). Recovered consciousness: A hypothesis concerning modularity and episodic memory. Journal of Clinical \& Experimental Neuropsychology, 17, 276-290.

Mulligan, N. W., \& Hartman, M. (1996). Divided attention and indirect memory tests. Memory \& Cognition, 24, 453-465.

Nelson, D. L., Canas, J. L., Bajo, M. T., \& Keelan, P. D. (1987). Comparing word fragment completion and cued recall with letter cues. Journal of Experimental Psychology: Learning, Memory, \& Cognition, 13, 542-552.

Nelson, D. L., Schreiber, T. A., \& McEvoy, C. L. (1992). Processing implicit and explicit representations. Psychological Review, 99, 322348.

Norman, D. A., \& Shallis, T. (1986). Attention to action: Willed and automatic control of behavior. In R. J. Davidson, G. E. Schwartz, \& D. Shapiro (Eds.), Consciousness and self-regulation: Advances in research and theory (pp. 1-18). New York: Plenum.

Parkin, A. J., ReID, T. K., \& Russo, R. (1990). On the differential nature of implicit and explicit memory. Memory \& Cognition, 18, 507514.

Patterson, K., \& Hodges, J. R. (1992). Deterioration of word meaning: Implications for reading. Neuropsychologia, 30, 1025-1040.

Posner, M. I., \& SNYDER, C. R. R. (1975). Attention and cognitive control. In R. L. Solso (Ed.), Information processing and cognition: The Loyola symposium (pp. 55-85). Hillsdale, NJ: Erlbaum.

Reingold, E. M., \& Goshen-Gottstein, Y. (1996). Separating consciously controlled and automatic influences in memory for new associations. Journal of Experimental Psychology: Learning, Memory, \& Cognition, 22, 397-406.

RichardSON-KLAVEhN, A., \& GARdiner, J. M. (1995). Retrieval volition and memorial awareness in stem completion: An empirical analysis. Psychological Research, 57, 166-178.

RichaRDSON-KLAVEHN, A., \& GARDINER, J. M. (1996). Cross-modal priming in stem completion reflects conscious memory, but not voluntary memory. Psychonomic Bulletin \& Review, 3, 238-244.

Richardson-Klavehn, A., \& Gardiner, J. M. (1998). Depth-ofprocessing effects on priming in stem completion: Tests of the voluntary-contamination, conceptual-processing, and lexicalprocessing hypotheses. Journal of Experimental Psychology: Learning, Memory, \& Cognition, 24, 593-609.

Richardson-Klavehn, A., Gardiner, J. M., \& JaVA, R. I. (1994). Involuntary conscious memory and the method of opposition.Memory, 2, 1-29.

Richardson-Klavehn, A., Lee, M. G., Joubran, R, \& Bjork, R. A. (1994). Intention and awareness in perceptual identification priming. Memory \& Cognition, 22, 293-312.

RoEdiger, H. L., III (1990). Implicit memory: Retention without remembering. American Psychologist, 45, 1043-1056.

Roediger, H. L., III, \& Blaxton, T. A. (1987a). Effects of varying modality, surface features, and retention interval on priming in wordfragment completion. Memory \& Cognition, 15, 379-388.

Roediger, H. L., III, \& Blaxton, T. A. (1987b). Retrieval modes produce dissociations in memory for surface information. In D. S. Gorfein \& R. R. Hoffman (Eds.), Memory and learning: The Ebbinghaus centennial conference (pp. 349-380). Hillsdale, NJ: Erlbaum.

Roediger, H. L., III, \& McDermott, K. B. (1993). Implicit memory in normal human subjects. In H. Spinnler \& F. Boller (Eds.), Handbook of neuropsychology (pp. 63-131). Amsterdam: Elsevier.

SCHACTER, D. L. (1992). Priming and multiple memory systems: Per- 
ceptual mechanisms of implicit memory. Journal of Cognitive Neuroscience, 4, 244-256.

Schacter, D. L., Bowers, J. [S.], \& Booker, J. (1989). Intention, awareness, and implicit memory: The retrieval intentionality criterion. In S. Lewandowsky, J. C. Dunn, \& K. Kirsner (Eds.), Implicit memory: Theoretical issues (pp. 47-65). Hillsdale, NJ: Erlbaum.

Schacter, D. L., \& Church, B. A. (1992). Auditory priming: Implicit and explicit memory for words and voices. Journal of Experimental Psychology: Learning, Memory, \& Cognition, 18, 915-930.

Schacter, D. L., \& Graf, P. (1986). Effects of elaborative processing on implicit and explicit memory for new associations. Journal of Experimental Psychology: Learning, Memory, \& Cognition, 12, 432-444.

Shimamura, A. P., Janowsky, J., \& SQuire, L. R. (1990). Memory for the temporal order of events in patients with frontal lobe lesions and amnesic patients. Neuropsychologia, 28, 803-813.

Shimamura, A. P., \& Squire, L. R. (1989). Impaired priming of new associations in amnesia. Journal of Experimental Psychology: Learning, Memory, \& Cognition, 15, 721-728.

Smith, S. M., \& Tindell, D. R. (1997). Memory blocks in word fragment completion caused by involuntary retrieval of orthographically related primes. Journal of Experimental Psychology: Learning, Memory, \& Cognition, 23, 355-370.

Tulving, E. (1983). Elements of episodic memory. Oxford: Oxford University Press, Clarendon Press.

Tulving, E. (1985). Memory and consciousness. Canadian Psychologist, 26, 1-12.

Tulving, E., \& Thomson, D. M. (1973). Encoding specificity and retrieval processes in episodic memory. Psychological Review, 80, 352372.

Vaidya, C. J., Gabrieli, J. D. E., Verfaellie, M., Fleischman, D., \& ASKARI, N. (1998). Font-specific priming following global amnesia and occipital lobe damage. Neuropsychology, 12, 183-192.

Whittlesea, B. W. A., \& Cantwell, A. L. (1987). Enduring influence of the purpose of experiences: Encoding-retrieval interactions in word and pseudoword perception. Memory \& Cognition, 15, 465472 .

Winocur, G., Moscovitch, M., \& Stuss, D. T. (1996). Explicit and implicit memory in the elderly: Evidence for double dissociation involving medial temporal- and frontal-lobe functions. Neuropsychology, 10, 57-65.

\section{NOTES}

1. Richardson-Klavehn and Gardiner $(1995,1996)$ reported that an on-line recognition measure in an implicit stem completion test showed a strong advantage of semantic over graphemic encoding, even though depth of processing had only a small influence on the size of priming effect. They used this finding to argue that awareness of the occurrence of the item in the study episode does not play a causal role in modulating the amount of repetition priming. Their argument is contrary to what I will argue shortly, and I offer two comments in response. The first is that the depth-of-processing effect on stem completion is always small numerically. What is important is that an effect is observed at all, contrary to what is expected from the position that stem completion is a data-driven task. Second, the depth-of-processing effect that they observed with the on-line recognition measure may not have reflected involuntary recognition but instead the intentional aspect of recognition, such as retrieval of context. Support for this claim can be found in the finding that depth of processing enhances remember responses but not know responses in a modified recognition task (Gardiner, 1988), in which subjects are required to respond remember when they can recollect the contextual detail in which the studied item was encountered, and respond know when they cannot. Thus, the depth-of-processing effects on a direct recognition measure and on repetition priming may not necessarily reflect the same mechanism.

2. Schacter and colleagues' (Schacter \& Church, 1992) results from an auditory stem completion task are also consistent with this pattern.

It should be noted that not all typography-specific priming effects reflect involuntary aware memory. Typography effects in data-driven implicit memory tests are also predicted from the TAP framework when the encoding task emphasizes data-driven operations (see, e.g., Curran et al., 1996). Also, data indicating that a specific visual form system located in the right hemisphere mediates the typography-specific priming effect (e.g., Marsolek, Kosslyn, \& Squire, 1992; Vaidya, Gabrieli, Verfaellie, Fleischman, \& Askari, 1998) are best interpreted outside the current involuntary aware memory framework.

3. New association priming effects have been found in amnesic patients in some studies (e.g., Cermak, Blackford, O'Connor, \& Bleich, 1988). For a more comprehensive description of the findings, readers are referred to Kinoshita (1999).

4. The idea that semantics have a binding property is not new, as can be seen in the "semantic glue" hypothesis proposed by Patterson and Hodges (1992) to explain the frequently observed co-occurrence of surface dyslexia (the inability to read words that do not follow the regular spelling-to-sound correspondence rule, such as yacht or pint) and impairment of semantic memory. Patterson and Hodges suggested that semantic comprehension plays an important role in binding together segments of phonological lexical codes: When semantic memory breaks down, the "semantic glue" that binds the phonological elements together also breaks down, and deficits appear in reading words that do not conform to the consistently occurring relationship between orthography and phonology at the subword level.

(Manuscript received January 5, 1999; revision accepted for publication April 13, 2000.) 